\title{
Methylation Status of the miR-141-3p Promoter Regulates miR-141-3p Expression, Inflammasome Formation, and the Invasiveness of HTR-8/SVneo Cells
}

\author{
Dongcai $\mathrm{Wu}^{\mathrm{a}} \mathrm{LiShi}^{\mathrm{b}}$ Fangrong Chen ${ }^{\mathrm{a}}$ Qing Lin ${ }^{\mathrm{a}}$ Jiao Kong ${ }^{\mathrm{a}}$ \\ aDepartment of Obstetrics, Hainan General Hospital, Hainan Affiliated Hospital of Hainan Medical University, \\ Haikou, PR China; bepartment of Medical Ultrasonics, Hainan General Hospital, Hainan Affiliated Hospital of \\ Hainan Medical University, Haikou, PR China
}

\section{Keywords}

Preeclampsia · Methylation · microRNA-141 · Proliferation · Invasion

\begin{abstract}
MicroRNA-141 (miR-141-3p) is upregulated in preeclampsia. This study investigated the effect of methylation of the miR141-3p promoter on cell viability, invasion capability, and inflammasomes in vitro. The expression of miR-141-3p and methylation status of the miR-141-3p promoter were examined by RT-qPCR and pyrosequencing in villus tissues of women with spontaneous delivery (VTsd), villus tissues of women with preeclampsia (VTpe), and also in HTR-8/SVneo cells treated with a miR-141-3p inhibitor and $20 \mu \mathrm{mol} / \mathrm{L}$ 5-aza-2'-deoxycytidine (5-Aza), a DNA methyltransferase inhibitor. Cell viability and invasion were evaluated by CCK-8 and transwell assays. In addition, the levels of CXCL12, CXCR4, CXCR2, MMPs, NLRP3, and ASC expression were assessed by western blotting, and IL-1 $\beta$ and IL-18 concentrations were assayed by ELISA. miR-141-3p expression was upregulated, and the levels of miR-141-3p promoter methylation and CXCL12, CXCR4, and CXCR2 expression were decreased in VTpe relative to VTsd. In HTR-8/SVneo cells, hypomethylation caused by 5 -Aza treatment increased miR-
\end{abstract}

141-3p expression, while DNA methyltransferase 3 (DNMT3) transfection decreased miR-141-3p expression. miRNA141-3p induced NLRP3, IL-1 $\beta$, and IL-18 production, decreased CXCR4, MMP, and MMP2 production, and suppressed cell growth and invasion. Furthermore, we observed that NLRP3 plays an important mediatory role in the effects of miR-141-3p described above. Decreased methylation of the miR-141-3p promoter increases miR-141-3p expression, which in turn increases NLRP3 expression, resulting in higher IL-1 $\beta$ and IL-18 levels and lower levels of MMP2/9 and CXCR4. We conclude that modification of the miR-141-3p promoter might be a curial mediator in preeclampsia.

(c) 2021 S. Karger AG, Basel

\section{Introduction}

Preeclampsia is a pregnancy-related multisystem disease that can result in multiple organ injury and severe fetal complications. It is one of the primary reasons for maternal and infant illness and death, and causes over 76,000 maternal and 500,000 perinatal deaths worldwide each year [Steegers et al., 2010]. Preeclampsia can cause

D.W. and L.S. contributed equally to this work. karger@karger.com

www.karger.com/cgr

Karger ${ }^{\prime \prime}=$ (c) 2021 S. Karger AG, Base

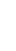


severe complications in pregnant women, such as eclampsia, acute renal failure, pulmonary edema, liver rupture, placental abruption or coagulation disorders [Croles et al., 2017]. Preeclampsia can also cause fetal growth restriction, intrauterine distress, and even stillbirth. In addition, pregnant women with severe preeclampsia are also at an increased risk for developing high blood pressure, kidney disease, and thrombogenesis in the future. A meta-analysis of approximately 35 million subjects found that women with a history of preeclampsia had a significantly increased risk for developing long-term cardiovascular disease, were at a 4-fold increased risk for developing hypertension, and nearly 2 -fold increased risks for ischemic heart disease, stroke, and venous thromboembolism [Bellamy et al., 2007]. Increasing evidence indicates that inflammation plays an important role in preeclampsia [Mulla et al., 2011]. Inflammasomes are activated by a host-derived danger-associated molecular pattern, which is recognized by pattern recognition receptors such as nucleotide-binding domain leucine-rich repeat containing proteins (NLRPs). As a member of the NLRP family, NLRP3 in trophoblasts has been demonstrated to contribute to placental inflammation in preeclampsia, to form an inflammasome, and facilitate IL-18 and IL-1 $\beta$ production [Stødle et al., 2018]. Trophoblasts are immune-competent cells, and their apoptosis and impaired invasion play an important role in the development of preeclampsia [Mulla et al., 2011]. However, this pathological mechanism needs to be further elucidated.

MicroRNAs (miRNA) comprise a class of noncoding single-stranded small RNA molecules that are widely distributed in human tissues [Mohr and Mott, 2015]. miRNAs contain 21-23 nucleotides and have the characteristics of high conservation, temporal expression, and tissue specificity [Pepin and Gantier, 2016]. miRNAs can induce the degradation of mRNA or inhibit its translation by specifically binding the $3^{\prime}$-untranslated regions $\left(3^{\prime}\right.$ UTRs) of their target gene's mRNA molecule, and thus regulate target gene expression at the post-transcriptional level [Ritchie, 2017], which enables them to participate in various types of diseases. In recent years, increasing numbers of studies have demonstrated the involvement of miRNA in the pathogenesis and development of preeclampsia [Jairajpuri and Almawi, 2016; McNally et al., 2017]. Our laboratory showed that miR-141-3p was induced in trophoblasts under conditions of hypoxia, and impaired trophoblast invasion and vascularization [Wu et al., 2019]. Therefore, we hypothesized that aberrant expression of miR-141-3p occurs and participates in preeclampsia.
Similar to various proteins, miRNA expression is regulated epigenetically, and one important method is to regulate the methylation of $\mathrm{CpG}$ islands in a gene promoter region. It has been reported that increased rates of miR-129-2 and miR-663a promoter methylation may contribute to marked decreases in miR-129-2 and miR663a expression and the subsequent development of urothelial carcinoma [Padrão et al., 2017]. The upstream events leading to miR-141-3p generation remain unclear. In the early stage of this study, we identified the CpG island in the miR-141-3p promoter region by performing a bioinformatics analysis, and speculated that methylation of that island might determine changes in miR-141$3 p$ expression.

In the present study, we investigated the expression levels miR-141-3p and the methylation status of the miR141-3p promoter in preeclampsia. We used 5-aza-2'deoxycytidine (5-Aza), a DNA methyltransferase inhibitor, and forced expression of DNMT3b to evaluate the role played by promoter methylation in miR-141-3p expression in HTR-8/SVneo cells, and subsequent effects on cell growth and motility. Furthermore, we explored the downstream pathway regulated by miR-141-3p.

\section{Materials and Methods}

\section{Tissue Specimens}

Villus tissue samples from women with preeclampsia (VTpe) were obtained from Hainan General Hospital between November 2016 and February 2018. All the women provided their informed consent for collection of villus tissue with full-thickness sections of placenta. Villus tissues from normotensive women who experienced a spontaneous delivery (VTsd) were collected as controls. Pregnant women with primary hypertension or a suspected perinatal infection were not included in the control group. Baseline characteristics of the patients are shown in Table 1. All tissue samples were collected and immediately frozen at $-80^{\circ} \mathrm{C}$ until use.

\section{Cell Culture and Transfection}

HTR-8/SVneo cells (No. BNCC337655) were provided by the BeNa Culture Collection (Kunshan, Jiangsu Province, China). HTR-8/SVneo cells were cultured in RPMI 1640 medium (SigmaAldrich, Saint Louis, MO, USA) supplemented with 10\% fetal bovine serum (Sigma-Aldrich) and $1 \%$ penicillin/streptomycin (Hyclone; cat. No. SV30010) at $37^{\circ} \mathrm{C}$ in an atmosphere consisting of $5 \% \mathrm{CO}_{2}$ and $95 \%$ air. The negative control (NC) and miR-141-3p inhibitor were purchased from GenePharma (Shanghai, China). HTR-8/SVneo cells were placed into 6 -well plates $\left(1 \times 10^{5}\right.$ cells/ well) and cultured for $8 \mathrm{~h}$; after which, they were transfected with $3.33 \mu \mathrm{g}$ of the NC, miR-141-3p mimics or inhibitor using Lipofectamine 3000 (Invitrogen, Carlsbad, CA, USA) according to the manufacturer's instructions. Other groups of HTR-8/SVneo cells were treated with $20 \mu \mathrm{mol} / \mathrm{L}$ 5-Aza (Sigma Aldrich) dissolved in DMSO. The medium containing 5-Aza was changed every $24 \mathrm{~h}$ 
Table 1. Clinical features of the patients involved in this study

\begin{tabular}{|c|c|c|c|c|c|}
\hline & Control $(n=20)$ & \multicolumn{3}{|l|}{ Preeclampsia } & $p$ value \\
\hline Age, years & $31.75 \pm 1.77$ & $30.70 \pm 1.98$ & $31.14 \pm 1.92$ & $29.67 \pm 1.86$ & $>0.05$ \\
\hline Systolic blood pressure, mmHg & $119.71 \pm 8.69$ & $167.32 \pm 5.83$ & $169.42 \pm 5.39$ & $162.40 \pm 3.49$ & $<0.01$ \\
\hline Diastolic blood pressure, $\mathrm{mmHg}$ & $76.44 \pm 4.21$ & $108.58 \pm 6.48$ & $109.39 \pm 6.70$ & $106.70 \pm 6.06$ & $<0.01$ \\
\hline
\end{tabular}

during a total period of $72 \mathrm{~h}$. DNMT3b cDNA was inserted into a pcDNA3.1 vector, which was then transfected into cells using Lipofectamine 3000, or co-transfected along with miR-141-3p or miR-141-3p + siRNA against NLRP3. The sequences used for NLRP3 siRNA and the corresponding control were UCUUAAAUUUCUUCAAGUCCAdTdT and GAUGCGAUACAGGUAAAUACAdTdT, respectively.

Reverse Transcription-Quantitative Real-Time PCR Assay

Total RNA was exacted from VTsd and VTpe or the treated HTR-8/SVneo cells by using TRIzol reagent (Invitrogen) as de-

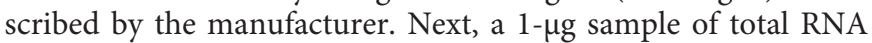
from each RNA extract was reverse transcribed into cDNA using a high-fidelity Reverse Transcription Kit (Applied Biosystems, Foster City, CA, USA). Subsequently, miR-141-3p expression was analyzed by qPCR performed using Bestar ${ }^{\mathrm{TM}}$ qPCR MasterMix (DBI Bioscience, Newark, DE, USA) on an ABI PRISM 7500 Sequence Detection System (Life Technologies, Carlsbad, CA, USA). The primers used in this study were as follows: U6 forward, CTCGCTTCGGCAGCACA; U6 reverse, AACGCTTCACGAATTTGCGT; all R, CTCAACTGGTGTCGTGGA; miR-141-3p reverse transcription primer, CTCAACTGGTGTCGTGGAGTCGGCAATTCAGTTGAGCCATCTT; miR-141-3p forward, ACACTCCAGCTGGGTAACACTGTCTGGTAAAG. The relative levels of gene expression were calculated using the $2^{-\Delta \Delta \mathrm{Ct}}$ method [Livak and Schmittgen, 2001], and U6 served as an endogenous control gene. All real-time reactions were repeated for 3 times in this study.

\section{Methylation Analysis}

Pyrosequencing analysis was used to determine the methylation levels of the miR-141-3p promoter regions in VTsd and VTpe or the treated HTR-8/SVneo cells. Similar to a previous study [Delaney et al., 2015], a bisulfite conversion process was performed using an Epitect Bisulfite Kit (Qiagen Technologies; cat. No. 59124 ) according to instructions provided by the supplier, and the CpG sites were examined using a PyroMark Q96 MD workstation (Qiagen, Venlo, Netherlands). Briefly, the PCR products were denatured with sodium hydroxide and then annealed by heating $\left(80^{\circ} \mathrm{C}\right.$ for $\left.2 \mathrm{~min}\right)$. After properly adjusting the PyroMark CpG software (Qiagen $\mathrm{GmbH}$; version 1.0), we mixed the enzyme with the substrate in $620 \mu \mathrm{L}$ of water. The reaction products were identified by sequencing, and the methylation levels were expressed as the average methylation percentage of $\mathrm{CpG}$ sites.

Methylation of the miR-141-3p Promoter

Serves to Inhibit Preeclampsia
Western Blot Assay

Total proteins were extracted using RIPA lysis and extraction buffer (Thermo Scientific, cat. No. 89900) supplemented with PMSF (Solarbio; cat. No. P0100). The total protein concentration in each extract was determined using a BCA Protein Assay Kit (Pierce, Rockford, IL, USA). Next, an equal amount of total protein from each sample was separated by $10 \%$ SDS-PAGE, and the separated protein bands were transferred onto PVDF membranes (Millipore, Burlington, MA, USA), which were subsequently blocked with $5 \%$ low fat-milk for $2 \mathrm{~h}$. After being blocked, the membranes were treated with primary antibodies at $4^{\circ} \mathrm{C}$ overnight, and then treated with a secondary antibody for $2 \mathrm{~h}$. The immunostained protein bands were visualized using enhanced chemiluminescent (ECL) reagents (Millipore), and results were determined using a HumaBlot 44FA system. The primary antibodies used were anti-CXCL12 (Abcam, Cambridge, UK; ab9797), anti-CXCR4 (Abcam; ab1670), anti-CXCR2 (Abcam; ab14935), anti-MMP2 (Abcam; ab37150), anti-MMP9 (Abcam; ab38898), anti-NLRP3 (Boster, Pleasanton, CA, USA; BA3677), anti-ASC (Abcam; ab180799), and anti-GAPDH (Abcam; ab9482). GAPDH was used as an internal reference standard.

\section{Bioinformatics Analysis}

The bioinformatics analysis of miR-141-3p was carried out using DAVID online database (https://david.abcc.ncifcrf.gov/). The target genes of miR-141-3p were obtained by Targetscan (http:// www.targetscan.org/vert_72/), and then GO analysis was performed in DAVID online database.

\section{Cell Viability}

Treated HTR-8/SVneo cells were resuspended in culture medium containing $10 \%$ PBS. Next, $100 \mu \mathrm{L}$ of suspended HTR-8/ SVneo cells were seeded into 96 -well plates $\left(5 \times 10^{3}\right.$ cells/well $)$ and cultured in a $37^{\circ} \mathrm{C}$ incubator containing $5 \% \mathrm{CO}_{2}$. After $0,24,48$, and $72 \mathrm{~h}$ of culture, $10 \mu \mathrm{L}$ of cell counting kit-8 solution (CCK-8; Dojindo, Japan) was added to the cells in each well, and the cells were incubated for an additional $3 \mathrm{~h}$. Next, the absorbance value (OD) of each well at $450 \mathrm{~nm}$ was measured using a SpectraMax microplate reader (Molecular Devices, San Jose, CA, USA).

Transwell Assay

Treated HTR-8/SVneo cells $\left(200 \mu \mathrm{L}, 3 \times 10^{6}\right.$ cells $\left./ \mathrm{mL}\right)$ were added into the upper chamber of a transwell plate (Corning Inc., Corning, NY, USA) that had been pre-coated with Matrigel (BD 

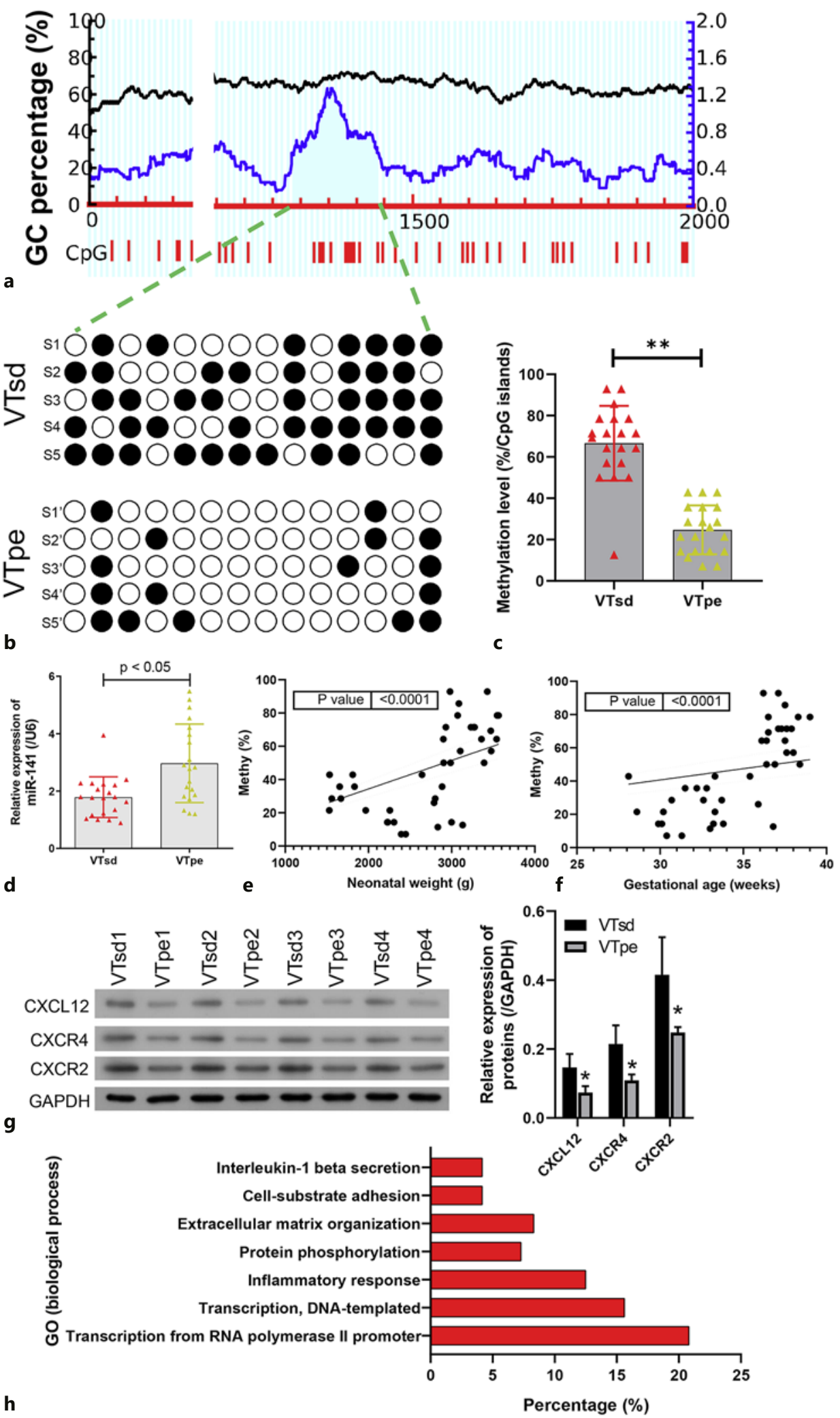

1

(For legend see next page.) 
Biosciences, Franklin Lakes, NJ, USA), and $600 \mu \mathrm{L}$ of culture medium containing $10 \%$ fetal bovine serum was added into the lower chamber. After incubation for $24 \mathrm{~h}$, the invasive cells were fixed with $4 \%$ paraformaldehyde (Solarbio; cat. No. P1110) and stained with $0.5 \%$ crystal violet (Sigma Aldrich). The results were observed using an inverted microscope.

\section{Enzyme-Linked Immunosorbent Assay}

The supernatants of cultured cells were collected for determination of IL- $1 \beta$ and IL-18 concentrations. The levels of cytokines in the supernatants were measured according to the instructions provided by the manufacturer of the ELISA kit.

\section{Immunofluorescence Staining}

Cultured cells were fixed with $4 \%$ formaldehyde for $10 \mathrm{~min}$ at room temperature. After permeabilization with $0.2 \%$ Triton $\mathrm{X}-100$, the cells were first incubated with anti-NLRP3 antibody for $1 \mathrm{~h}$ at room temperature, and then incubated with a secondary antibody and DAPI (Beyotime, Nantong, China) for $1 \mathrm{~h}$ at room temperature. Representative photographs of immunofluorescence staining results were obtained using a confocal laser scanning microscope (LSM510; Zeiss, Germany).

\section{Statistical Analysis}

Differences between groups were analyzed via the Student $t$ test performed using IBM SPSS Statistics for Windows, Version 20 software (IBM Corp., Armonk, NY, USA). Results are presented as mean \pm SD obtained from at least 3 independent experiments. A $p$ value $<0.05$ was considered to be statistically significant.

\section{Results}

VTpe Showed Upregulation of miR-141-3p Expression and Hypomethylation of the miR-141-3p Promoter

To investigate the potential regulatory mechanism of miR-141-3p expression, the miR-141-3p promoter sequence was analyzed by a bioinformatics analysis. Those results showed there was a CpG island in the miR-141-3p promoter region (Fig. 1a). Next, we performed a pyrose-

Fig. 1. Expression and methylation status of the miR-141-3p promoter in VTsd and VTpe. a miR-141-3p promoter sequences were predicted using UCSC. A CpG island existed in the promoter region (-2000, shown in light blue). $\mathbf{b}$ The methylation status of the miR-141-3p promoter in VTsd $(n=20)$ and VTpe $(n=20)$ was evaluated by the BSP assay. Black dots indicate methylation and white dots indicate non-methylation. $\mathbf{c}$ The methylation levels of miR-141-3p in VTsd $(n=20)$ and VTpe $(n=20)$ were examined using RT-qPCR assays; $p<0.05$. $\mathbf{d}$ Difference in expression of miR$141-3 p$ in VTsd and VTpe; $p<0.05$. e, $\mathbf{f}$ Correlation between neonatal weight (e) or gestational age (f) and methylation level of miR141-3p promoter. $\mathbf{g}$ Western blotting analyses of CXCL12, CXCR4, and CXCR2 levels in VTsd and VTpe. GAPDH served as an internal reference. $\mathbf{h}$ Bioinformatics analysis of target genes of miR141-3p.

Methylation of the miR-141-3p Promoter

Serves to Inhibit Preeclampsia quencing assay to examine the methylation status of the miR-141-3p promoter in 20 VTsd and 20 VTpe. The results showed that the methylation status of the miR-141$3 p$ promoter was lower in the VTpe than in the VTsd (Fig. 1b, c). Furthermore, RT-qPCR assays revealed that the miR-141-3p levels in VTpe were significantly higher $(p<0.05)$ than those in VTsd (Fig. 1d). Also, a positive correlation was found between neonatal weight and methylation level of the miR-141-3p promoter as well as gestational age and methylation level of miR-141-3p promoter (Fig. 1e, f). Furthermore, our results showed that the levels of CXCL12, CXCR4, and CXCR2 protein were dramatically downregulated in VTpe relative to those in VTsd (Fig. 1g). Further bioinformatics analysis showed that the target gene of miR-141-3p might participate in biological processes, including inflammatory response, extracellular matrix organization, interleukin $1 \beta$ secretion (Fig. 1h).

\section{miR-141-3p Played an Important Role in Villous Trophoblast Motility}

To examine the role played by $\mathrm{miR}-141-3 \mathrm{p}$ in the invasion of villous trophoblasts, HTR-8/SVneo cells were transfected with either mimics and/or inhibitors. As shown in Figure 2a, transfection with the miR-141-3p mimics and inhibitors significantly increased and decreased the levels of miRNA in the cells, respectively, indicating that the transfections had been successfully established. As expected, the effect on cell invasion varied, as shown by a significant increase and decrease after transfection with the inhibitor and mimics, respectively (Fig. 2b). While, we also found that inhibitor of miR-141$3 p$ and mimics of miR-141-3p reversed the effect of mimics and inhibitor, respectively (Fig. 2b). Concurrently, cell viability showed a consistent increase after miR-141-3p knockdown and a decrease after miR-141-3p overexpression (Fig. 2c). ELISA examinations showed that IL-18 and IL-1 $\beta$ levels were significantly increased in the cells transfected with miR-141-3p mimics (Fig. 2d, e). We also noticed a significant increase in NLRP3 expression and decreases in CXCR4, MMP2, and MMP9 expression in cells that overexpressed miR-141-3p (Fig. 2f). On the other hand, the levels of ASC expression were similar in each group. These results indicated that miR-141-3p regulated the expression of IL-18, IL-1 $\beta$, NLRP3, CXCR4, MMP2, and MMP9.

\section{Inhibition of miR-141-3p Promoter Methylation}

Enhanced the Mobility of Villous Trophoblasts

To investigate the role of promoter methylation in miR-141-3p expression, HTR-8/SVneo cells were ex- 

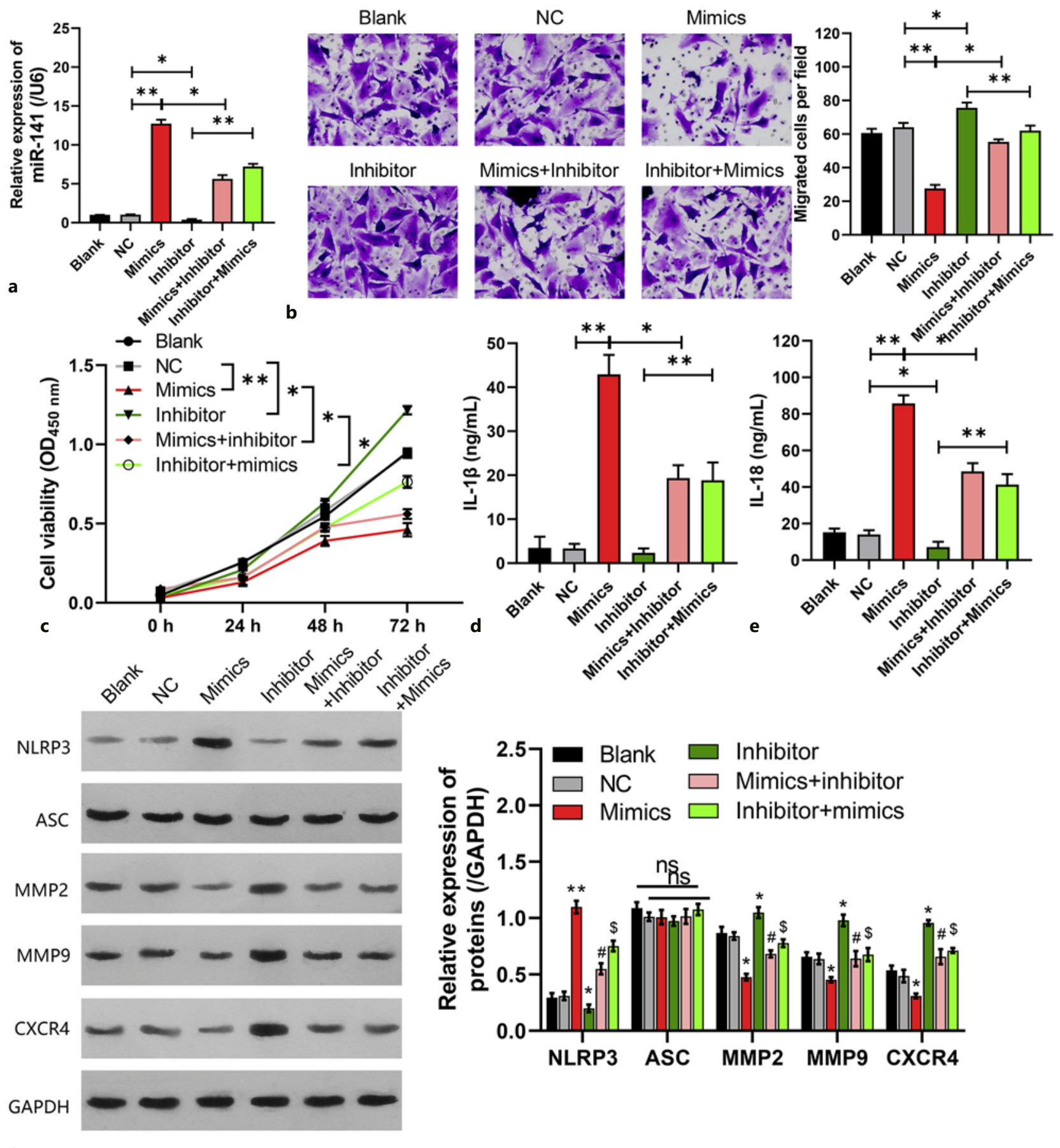

Fig. 2. miR-141-3p regulates $\mathrm{HTR}-8 / \mathrm{SV}$ neo cell invasion and growth. HTR-8/Svneo cells were transfected with the miR-141-3p inhibitor or mimics for $24 \mathrm{~h}$. a miR-141-3p expression in the parent and transfected cells was determined by RT-qPCR. b Cell invasion capability was determined by the transwell assay. Magnification, $\times 200$. c Cell viability was determined by the CCK- 8 assay. d, e The levels of IL-1 $\beta$ (d) and IL-18 (e) were determined by ELISA. $\mathbf{f}$ The levels of NLRP3, ASC, MMP2, CXCR4, and MMP9 expression were determined by western blotting. ${ }^{*} p<0.05$; ${ }^{* *} p<$ 0.01 , versus NC group or blank group. ${ }^{\#} \mathrm{p}<0.05$, versus inhibitor. $\$ \mathrm{p}<0.05$, versus mimics. 


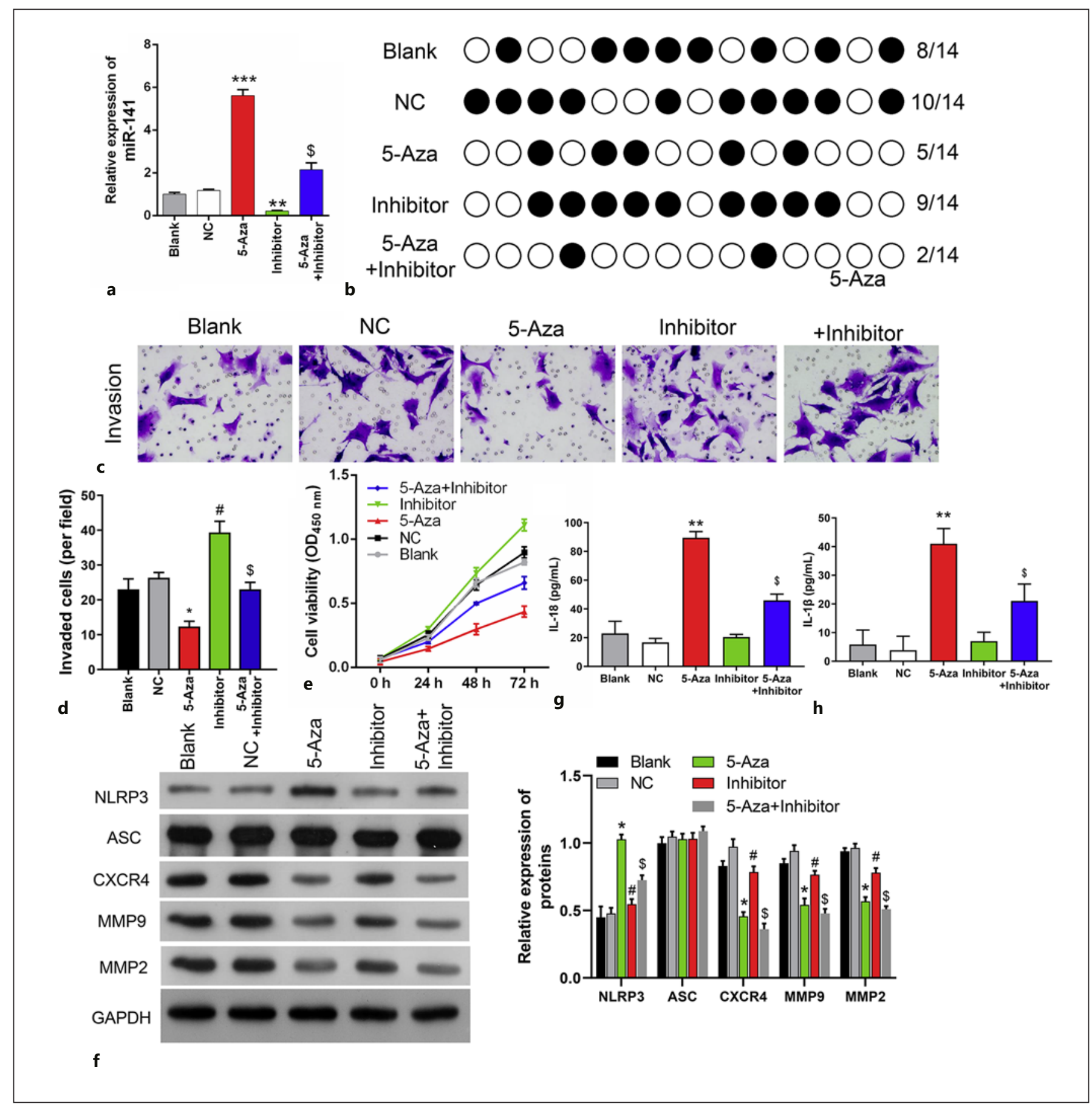

Fig. 3. Methylation-related inhibition of miR-141-3p accelerated the proliferation and invasion of HTR-8/SVneo cells. HTR-8/SVneo cells were treated with the miR-141-3p inhibitor, with $5 \mu \mathrm{M}$ 5-Aza alone, or with a combination of both for $24 \mathrm{~h}$. a miR-141-3p expression was determined by RT-qPCR. $\mathbf{b}$ The methylation status of the miR-141-3p promoter was evaluated by the BSP assay. c, d Cell invasion capability was determined by the transwell assay. e Cell viability was determined by the CCK- 8 assay. $\mathbf{f}$ The levels of NLRP3, ASC, CXCR4, MMP9, and MMP2 expression were determined by western blotting. $\mathbf{g}, \mathbf{h}$ The levels of IL-18 (g) and IL-1 $\beta$ (h) were determined by ELISA. ${ }^{*} p<0.05,{ }^{* *} p<0.01,{ }^{* * *} p<0.001$, versus NC group or Blank group; ${ }^{\$} p<0.05$, versus 5 -Aza group; ${ }^{\#} \mathrm{p}<0.05$, versus NC. 


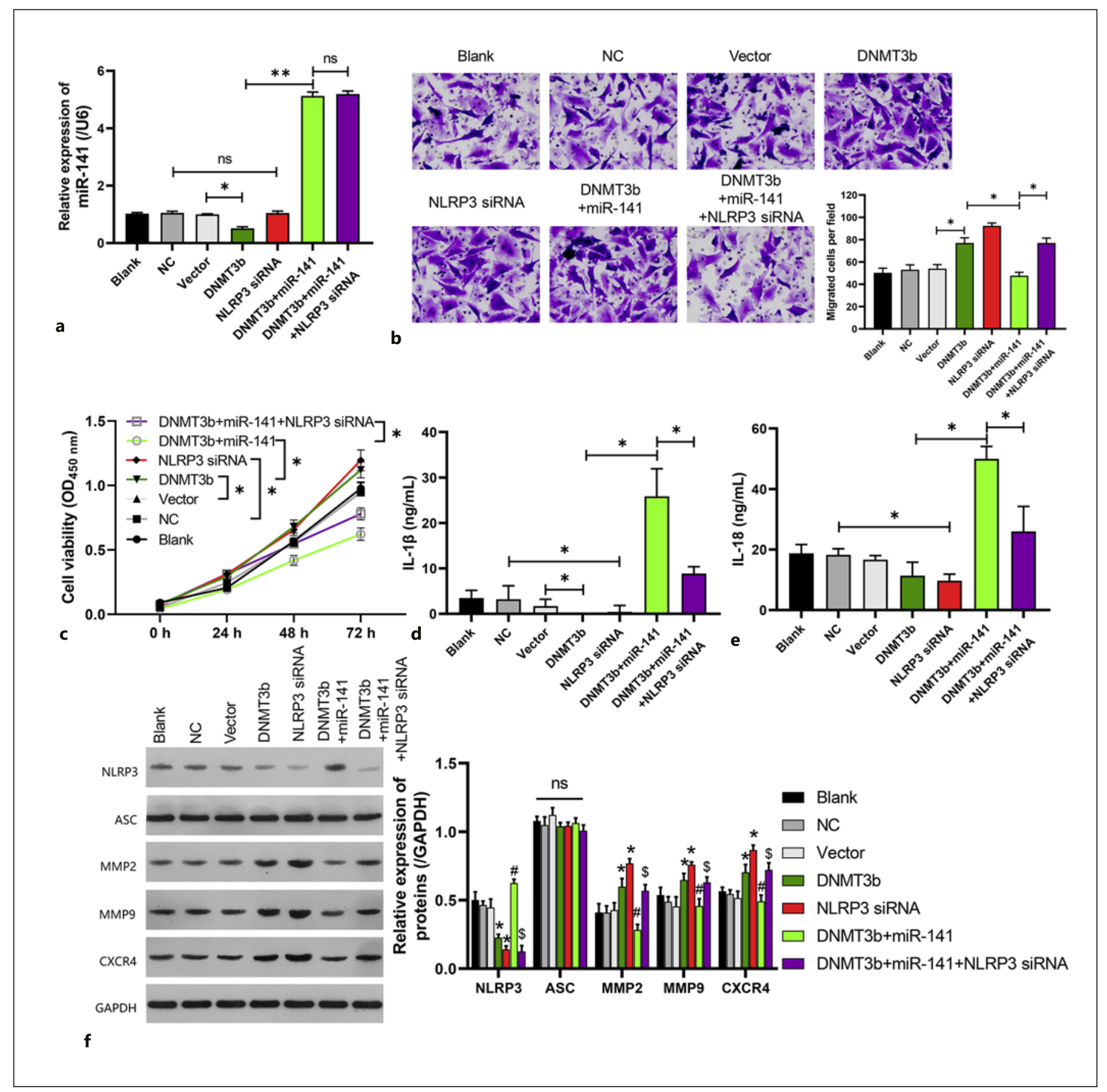

Fig. 4. NLRP3 mediated the inhibitory effect of miR-141-3p on the proliferation and invasion of HTR-8/SVneo cells. HTR-8/SVneo cells were transfected with DNMT3b or NLRP3 siRNA alone, or in combination with miR-141-3p or miR-141-3p plus siRNA against NLRP3 (NLRP3 siRNA) for $24 \mathrm{~h}$. a The levels of miR-141$3 \mathrm{p}$ expression were determined by RT-qPCR. $\mathbf{b}$ Cell invasion ca- pability was determined by the transwell assay. c Cell viability was determined by the CCK- 8 assay. d The levels of IL- $1 \beta$ (d) and IL-18 (e) were determined by ELISA. f The levels of NLRP3, ASC, CXCR4, MMP9, and MMP2 expression were determined by western blotting. ${ }^{*} p<0.05,{ }^{* *} p<0.01$, versus NC group or blank group; ${ }^{\$} p<0.05$, versus 5 -Aza group; ${ }^{\#} \mathrm{p}<0.05$, versus DNMT3b. 
Fig. 5. Schematic overview of the regulation and role of miR-141-3p in trophoblast proliferation and invasion. Downregulation of methylation in a CpG island in the miR-141-3p promoter region enhanced miR-141-3p expression. As a result, inflammasomes were induced, MMP2/9 expression, trophoblast proliferation and invasion were inhibited.

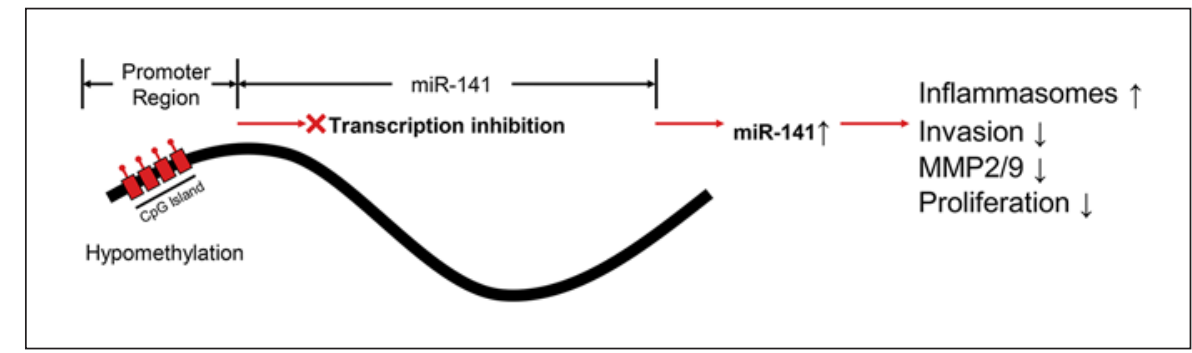

posed to 5-Aza. After 5-Aza treatment, there was a significant increase $(p<0.001)$ in miR-141-3p levels (Fig. 3a), and a consistent decrease in methylation of the miR-1413 p promoter (Fig. 3b), indicating that promoter methylation participated in regulating miR-141-3p expression. In cells transfected with the miR-141-3p inhibitor, there was a significant increase in miR-141-3p expression $(p<0.05$; $p<0.01)$ irrespective of whether the cells had been treated with 5-Aza (Fig. 3a). As expected, 5-Aza significantly suppressed the invasion capability of HTR-8/SVneo cells, while the miR-141-3p inhibitor dramatically enhanced the invasion capability of HTR-8/SVneo cells $(p<0.05$; $p<0.01$ ) (Fig. 3c, d). These data indicated that 5-Aza exposure attenuated cell motility via hypomethylation of the miR-141-3p promoter, thereby increasing miR-141$3 p$ expression. Similarly, 5-Aza markedly reduced the numbers of viable cells, and the reduction was inhibited by the miR-141-3p inhibitor, indicating that 5-Aza regulated cell viability by regulating miR-141-3p expression ( $p$ $<0.05, p<0.01$ ) (Fig. 3e). Additionally, a western blot analysis showed that 5-Aza treatment induced NLRP3 expression, downregulated CXCR4, MMP2, and MMP9, expression, but did not affect ASC expression (Fig. 3f). Similar to NLRP3, the levels of IL-18 and IL- $1 \beta$ were significantly increased $(p<0.01)$ after 5-Aza exposure (Fig. 3g, h).

\section{Roles of DNMT3b and NLRP3 in the Effect of miR-}

\section{1-3p on Villous Trophoblast Motility}

To investigate the mechanism by which inhibition of miR-141-3p affects cell motility, trophoblast cells were transfected with empty vector/DNMT3b, and NLRP3 siRNA NC and siRNA alone, or in combination with miR-141-3p and/or siRNA against NLRP3. It was observed that DNMT3b transfection and NLRP3 siRNA did not affect miR-141-3p expression (Fig. 4a). On the other hand, cell invasion capability and viability were increased after DNMT3b transfection and NLRP3 siRNA, and those effects were reversed by co-transfection with miR141-3p. Interestingly, the reversals in invasiveness and vi-

Methylation of the miR-141-3p Promoter

Serves to Inhibit Preeclampsia ability were significantly suppressed by additional transfection with si-NLRP3 (Fig. 4b, c). These data indicated that DNMT3b or NLRP3 knockdown enhanced cellular activity by inhibiting miR-141-3p, while NLRP3 appeared to negatively regulate cell activity irrespective of miR141-3p levels. Subsequent examinations showed that IL$1 \beta$ and IL-18 levels were not affected by DNMT3b transfection and NLRP3 siRNA, but were markedly increased after additional transfection with miR-141-3p; however, those increases were significantly suppressed after further transfection with si-NLRP3 $(p<0.01, p<0.05)$ (Fig. $4 \mathrm{~d}$, e). Western blot assays showed that the level of NLRP3 was decreased by DNMT3b transfection and NLRP3 siRNA and then was reversed by miR-141-3p. However, expression of MMP2, MMP9, and CXCR4 was not altered by DNMT3b. Further, we found that miR-141-3p induced elevation of NLRP3 and suppression of MMP2, MMP9, and CXCR4, which was then reversed by NLRP3 siRNA (Fig. 4f). When taken together, these results suggest that DNMT3b regulates miR-141-3p expression, which in turn regulates NLRP3 expression, and ultimately inflammasome.

\section{Discussion}

miRNAs comprise a class of noncoding RNAs that are widely involved in regulating post-transcriptional gene expression [Morales et al., 2017]. Studies have shown that miRNAs participate in cell differentiation, proliferation, apoptosis, and other important physiological and pathological processes [Cui J et al., 2017; Kim et al., 2017]. Currently, a number of miRNAs have been found to be differentially expressed in human and mouse placentas [Luo et al., 2017; Skalis et al., 2019]. In addition, several circulating miRNAs have been demonstrated to be the predictors of preeclampsia [Munaut et al., 2016; Jairajpuri et al., 2017]. Moreover, numerous miRNAs including miR4421 [Gao et al., 2018], miR-34a [Liu et al., 2019], miR517-5p [Fu et al., 2018], miR-182-5p [Fang et al., 2018], 
miR-195 [Wu et al., 2016], and miR-616-3p [Xu et al., 2018] might regulate the progression of preeclampsia. Therefore, aberrant expression of miRNAs might be involved in the development and pathological process of preeclampsia. In this study, we found that miR-141-3p was upregulated in VTpe when compared to VTsd, and such upregulation was due to decreased levels of miR141-3p promoter methylation. Furthermore, it was revealed for the first time that miR-141-3p regulated NRLP3 expression and its associated inflammatory responses, eventually resulting in reductions in the growth and invasion capabilities of HTR-8/SVneo cells.

Preeclampsia is a complex process involving multiple genes, factors, and stages [Helmo et al., 2018]. In recent years, more and more researchers have begun to devote themselves to the study of epigenetics, so as to clarify its mechanism in the physiological and pathological progression of diseases. Researches have shown that epigenetic mechanisms mainly involve DNA methylation, miRNA activity, and gene imprinting [Edwards et al., 2017]. Among those mechanisms, DNA methylation has attracted extensive attention from researchers in epigenetics. In mammals, DNA methylation mainly occurs on the $5^{\prime}$-terminal cytosine of a CpG dinucleotide, and involves the transfer of a methyl group to a specified DNA base site via the action of DNA methyltransferases (DNMTs) [Moore et al., 2013]. Studies have verified that abnormalities of DNA methylation are involved in several disease processes, including cancer [Kulis and Esteller, 2010; Li et al., 2016], psychiatric disorders [Liu et al., 2018], and autoimmune diseases [Sun et al., 2016]. Moreover, studies have also confirmed that trophoblast differentiation is related to the methylated and non-methylated states of DNA [Branco et al., 2016; Novakovic et al., 2017a, b]. Methylation of a $\mathrm{CpG}$ island in a gene promoter region can participate in preeclampsia pathogenesis by affecting the physiological function of trophoblast cells [Yeung et al., 2016; Mayne et al., 2017]. Numerous studies have suggested that gene silencing is associated with the abnormal methylation of a CpG island in the human genome. Studies have also confirmed that hypermethylation of miRNA promoter regions contributes to a downregulation of miRNA expression [Chhabra, 2015; Morales et al., 2017]. Neves et al. [2010] discovered that miR-200c/141 promoter activity could be repressed by DNA methylation, and that methylation of DNA in the miR-200c/141 promoter was strongly correlated with phenotype and the invasiveness of human breast cancer cell lines. Moreover, Pan et al. [2018] suggested that methylation of the miR141-3p promoter mediated the invasion and metastasis of
PDAC cells. In our study, we verified that the methylation level of the miR-141-3p promoter in VTpe was lower than that in VTsd, and was responsible for an increased level of miR-141-3p expression, which eventually led to decreased cell growth and motility. Additionally, our data revealed that DNMT3b might be associated with methylation of the miR-141-3p promoter in vitro. However, the exact mechanism of miR-141-3p promoter hypomethylation in vivo still needs to be confirmed.

Inflammatory cytokines function as crucial mediators in ovulation, implantation, placentation, and parturition during pregnancy [Saji et al., 2000]. The levels of pro-inflammatory cytokines in blood samples from preeclamptic women are significantly increased, suggesting that those increased cytokine levels contribute to trophoblastic apoptosis, intrauterine growth retardation, and eventually preeclampsia. In this study, we observed that miR141-3p induced the expression of NLRP3, most likely in an indirect manner. NLRP3 facilitated the formation of inflammasomes containing ASC and induced harmful inflammation in women with preeclampsia. IL- $1 \beta$ and IL18 are the pro-inflammatory cytokines activated by NLRP3, and their levels are significantly increased in the serum of women with preeclampsia [Mulla et al., 2011; Stødle et al., 2018]. Increased IL- $1 \beta$ levels are involved in the pathogenesis of preeclampsia, IL-18 can potentially enhance Fas ligand-mediated cytotoxicity, which is increased in preeclampsia and regulates placental apoptosis [Dong and Yin, 2014]. All these factors indicate that miR141-3p might be associated with preeclampsia by regulating NLRP3, IL-1 $\beta$, and IL-18 expression. In this study, we found that miR-141-3p inhibitor and mimics reversed the effect of mimics and inhibitor, respectively, on cell invasion, viability, IL- $1 \beta$ and IL-18 level, expression of NLRP3, MMP2/9, and CXCR4. These results suggested that miR141-3p functioned upstream of NLRP3 and CXCRs.

CXCL12 is a homeostasis/inflammatory chemokine that has chemotaxis for endothelial precursor cells, monocytes/macrophages, and B and T lymphocytes [Meng et al., 2018]. CXCR4, as the receptor for CXCL12, is a G protein-coupled receptor (GPCR, seven-pass transmembrane protein) composed of 352 amino acids [Pozzobon et al., 2016]. CXCL2, as a protein encoded by a protooncogene, can promote the formation of blood vessels and significantly influence the occurrence, development, and metastasis of tumors [Ha et al., 2017]. Studies have verified that the CXCL12/CXCL4/CXCL2 signaling pathway is involved in several key processes in human pregnancy, such as placental implantation and embryogenesis during early pregnancy, maternal-fetal immune toler- 
ance, and vascular remodeling [Sleightholm et al., 2017; Xue et al., 2017]. CXCL12 is mainly derived from placental trophoblast cells, including trophoblast progenitor cells, syncytiotrophoblasts, and extravillous trophoblast cells [Quinn et al., 2016]. CXCL12-mediated signals can regulate the differentiation of trophoblast progenitors into trophoblast subsets with different functions, and CXCL12 can promote the differentiation, invasion, and proliferation of trophoblast cells [Wang et al., 2015]. Additionally, signaling pathways, including CXCL12 $\beta$, MAPK1/ERK2, can also be mediated by miR-141-3p. Our previous study suggested that miR-141 mediated the trophoblast injured by hypoxia, and inhibition of miR-141 attenuated cell death, and promoted vascularization by targeting CXCL12 $\beta$ [Wu et al., 2019]. In the present study, we found that the levels of CXCL12, CXCR4, and CXCR2 expression were downregulated in VTpe compared to those in VTsd, and miR-141-3p inhibited CXCR4 expression. Besides, chemokines are widely regulated by other factors, including inflammatory responses mediated by NLRP3. Bitto et al. [2014] found that inhibition of the NLRP3 inflammasome in diabetic mice increased the expression of CXCL12, indicating that NLRP3 has the potential function to regulate CXCL12-CXCR4. But on the other hand, CXCL12-CXCR4 might be a mediator to regulate the NLRP3 inflammasome. Studies by Zendedel et al. [2016] in spinal cord injury showed that the expression of NLRP3-mediated inflammatory factors was generally upregulated after treatment with CXCL12. Therefore, we suspected that there is a feedback loop between NLRP3 and CXCL12/CXCR4, but it still needs further experimental exploration and verification.

Matrix metalloproteinases (MMPs) comprise a family of proteolytic enzymes that can hydrolyze almost all extracellular matrices [Cui $\mathrm{N}$ et al., 2017]. As important members of the family, MMP2 and MMP9 play an essential role in the invasion of trophoblast cells into endometrium [Chen and Khalil, 2017]. Therefore, MMP2 and MMP9 are the key hydrolytic enzymes required for trophoblast invasion. Previous studies have shown that MMP2 and MMP9 are closely related to preeclampsia [Feng et al., 2017; Laskowska, 2017; Sahay et al., 2018]. In our study, we showed that demethylation-mediated upregulation of the miR-141-3p promoter by 5-Aza treatment could downregulate MMP2 and MMP9 expression in HTR-8/ SVneo cells. Also, we found that knockdown of NLRP3 induced recovery of MMP2/9. The study of Unamuno et al. [2021] on obese tissues showed that the expression of genes related to extracellular matrix remodeling (such as $M M P 2)$ also decreased after inhibition of NLRP3, indicat-

Methylation of the miR-141-3p Promoter

Serves to Inhibit Preeclampsia ing that NLRP3 and MMP2 have a synergistic effect in obese tissues. Similarly, the relationship between NLRP3 and extracellular matrix-related factors (MMPs, COLs, etc.) is also similar in hepatic fibrosis and renal fibrosis [Dwivedi and Jena, 2018; Zhang et al., 2020]. In this study, knockdown of NLRP3 can promote the expression of extracellular matrix (MMP2, MMP9), indicating that there may be another way of regulation of NLRP3.

\section{Conclusion}

In summary, we found that miR-141-3p was upregulated in VTpe compared with VTsd, and this upregulation was characterized by lower levels of methylation in the miR-141-3p promoter region and lower levels of CXCL12, CXCR4, and CXCR2 expression. Our data revealed that hypomethylation of the miR-141-3p promoter increased miR-141-3p expression, which in turn induced inflammasome formation, decreased MMP2/9 expression, and inhibited trophoblast proliferation and invasion (Fig. 5). We conclude that miR-141-3p expression mediated by its promoter's methylation is related to the invasiveness of HTR-8/SVneo cells. The miR-141-3p promoter might serve as a target in preeclampsia therapy by altering its degree of methylation. However, only 20 patients and 20 control subjects were included in this study, and analysis of a larger cohort is expedient to corroborate our data. Besides, the specific mechanism of miR-141 mediating NLRP3 inflammasome was not explored and this is worth investigation.

\section{Statement of Ethics}

All procedures involving human participants were in accordance with the 1964 Helsinki declaration and its later amendments or comparable ethical standards. All patients provided their written informed consent for participation. The study protocol was approved by the Ethics Committee of Hainan General Hospital (Med-Eth-Re[2019]52).

\section{Conflict of Interest Statement}

The authors have no potential conflicts of interest to declare.

\section{Funding Sources}

This work was supported by Hainan Provincial Natural Science Foundation of China (No. 817306). 


\section{Author Contributions}

Conceptualization: Dongcai Wu. Methodology: Dongcai Wu. Formal analysis and investigation: Dongcai Wu, Fangrong Chen, Li Shi, and Qing Lin. Writing - original draft preparation: Dongcai $\mathrm{Wu}$ and Jiao Kong. Writing - review and editing: Dongcai Wu and Jiao Kong. Funding acquisition: Dongcai Wu. Resources: Dongcai Wu. Supervision: Jiao Kong. All authors commented on previous versions of the manuscript. All authors read and approved the final manuscript.

\section{Data Availability Statement}

All data generated or analyzed during this study are included in this article. Further enquiries can be directed to the corresponding author.

\section{References}

Bellamy L, Casas JP, Hingorani AD, Williams DJ. Pre-eclampsia and risk of cardiovascular disease and cancer in later life: systematic review and meta-analysis. BMJ. 2007;335:974.

Bitto A, Altavilla D, Pizzino G, Irrera N, Pallio G, Colonna MR, et al. Inhibition of inflammasome activation improves the impaired pattern of healing in genetically diabetic mice. $\mathrm{Br}$ J Pharmacol. 2014;171:2300-7.

Branco MR, King M, Perez-Garcia V, Bogutz AB, Caley M, Fineberg E, et al. Maternal DNA Methylation Regulates Early Trophoblast Development. Dev Cell. 2016;36:152-63.

Chen J, Khalil RA. Matrix Metalloproteinases in Normal Pregnancy and Preeclampsia. Prog Mol Biol Transl Sci. 2017;148:87-165.

Chhabra R. miRNA and methylation: a multifaceted liaison. Chembiochem. 2015;16:195203.

Croles FN, Nasserinejad K, Duvekot JJ, Kruip MJ, Meijer K, Leebeek FW. Pregnancy, thrombophilia, and the risk of a first venous thrombosis: systematic review and bayesian metaanalysis. BMJ. 2017;359:j4452.

Cui J, Zhou B, Ross SA, Zempleni J. Nutrition, microRNAs, and Human Health. Adv Nutr. 2017;8:105-12.

Cui N, Hu M, Khalil RA. Biochemical and Biological Attributes of Matrix Metalloproteinases. Prog Mol Biol Transl Sci. 2017;147:1-73.

Delaney C, Garg SK, Yung R. Analysis of DNA Methylation by Pyrosequencing. Methods Mol Biol. 2015;1343:249-64.

Dong W, Yin L. Expression of lipoxin A4, TNFa and IL-1 $\beta$ in maternal peripheral blood, umbilical cord blood and placenta, and their significance in pre-eclampsia. Hypertens Pregnancy. 2014;33:449-56.

Dwivedi DK, Jena GB. Glibenclamide protects against thioacetamide-induced hepatic damage in Wistar rat: investigation on NLRP3, MMP-2, and stellate cell activation. Naunyn Schmiedebergs Arch Pharmacol. 2018;391: 1257-74.

Edwards JR, Yarychkivska O, Boulard M, Bestor TH. DNA methylation and DNA methyltransferases. Epigenetics Chromatin. 2017;10: 23.
Fang YN, Huang ZL, Li H, Tan WB, Zhang QG, Wang L, et al. Highly expressed miR-182-5p can promote preeclampsia progression by degrading RND3 and inhibiting HTR-8/SVneo cell invasion. Eur Rev Med Pharmacol Sci. 2018;22:6583-90.

Feng H, Wang L, Zhang M, Zhang Z, Guo W, Wang X. Ratio of matrix metalloproteinase-2 to -9 is a more accurate predictive biomarker in women with suspected pre-eclampsia. Biosci Rep. 2017;37:BSR20160508.

Fu JY, Xiao YP, Ren CL, Guo YW, Qu DH, Zhang $\mathrm{JH}$, et al. Up-regulation of miR-517-5p inhibits ERK/MMP-2 pathway: potential role in preeclampsia. Eur Rev Med Pharmacol Sci. 2018;22:6599-608.

Gao X, Li H, Wei JX. MiR-4421 regulates the progression of preeclampsia by regulating $\mathrm{CY}$ P11B2. Eur Rev Med Pharmacol Sci. 2018;22: 1533-40.

Ha H, Debnath B, Neamati N. Role of the CXCL8CXCR1/2 Axis in Cancer and Inflammatory Diseases. Theranostics. 2017;7:1543-88.

Helmo FR, Lopes AMM, Carneiro ACDM, Campos CG, Silva PB, Dos Reis Monteiro MLG, et al. Angiogenic and antiangiogenic factors in preeclampsia. Pathol Res Pract. 2018;214:714.

Jairajpuri DS, Almawi WY. MicroRNA expression pattern in pre-eclampsia (Review). Mol Med Rep. 2016;13:2351-8.

Jairajpuri DS, Malalla ZH, Mahmood N, Almawi WY. Circulating microRNA expression as predictor of preeclampsia and its severity. Gene. 2017;627:543-8.

Kim D, Chang HR, Baek D. Rules for functional microRNA targeting. BMB Rep. 2017;50: 554-9.

Kulis M, Esteller M. DNA methylation and cancer. Adv Genet. 2010;70:27-56.

Laskowska M. Altered Maternal Serum Matrix Metalloproteinases MMP-2, MMP-3, MMP 9, and MMP-13 in Severe Early- and Late-Onset Preeclampsia. Biomed Res Int. 2017;2017: 6432426.

Li X, Zhu L, Ye X. [Aberrant DNA methylation and its targeted therapy in acute myeloid leukemia]. Zhejiang Da Xue Xue Bao Yi Xue Ban. 2016;45:387-94.

Liu C, Jiao C, Wang K, Yuan N. DNA Methylation and Psychiatric Disorders. Prog Mol Biol Transl Sci. 2018;157:175-232.
Liu JJ, Zhang L, Zhang FF, Luan T, Yin ZM, Rui $\mathrm{C}$, et al. Influence of miR-34a on preeclampsia through the Notch signaling pathway. Eur Rev Med Pharmacol Sci. 2019;23:923-31.

Livak KJ, Schmittgen TD. Analysis of relative gene expression data using real-time quantitative PCR and the 2(-Delta Delta C(T)) method. Methods. 2001;25:402-8.

Luo S, Cao N, Tang Y, Gu W. Identification of key microRNAs and genes in preeclampsia by bioinformatics analysis. PLoS One. 2017;12: e0178549.

Mayne BT, Leemaqz SY, Smith AK, Breen J, Roberts CT, Bianco-Miotto T. Accelerated placental aging in early onset preeclampsia pregnancies identified by DNA methylation. Epigenomics. 2017;9:279-89.

McNally R, Alqudah A, Obradovic D, McClements L. Elucidating the Pathogenesis of Preeclampsia Using In Vitro Models of Spiral Uterine Artery Remodelling. Curr Hypertens Rep. 2017;19:93.

Meng W, Xue S, Chen Y. The role of CXCL12 in tumor microenvironment. Gene. 2018;641: $105-10$.

Mohr AM, Mott JL. Overview of microRNA biology. Semin Liver Dis. 2015;35:3-11.

Moore LD, Le T, Fan G. DNA methylation and its basic function. Neuropsychopharmacology. 2013;38:23-38.

Morales S, Monzo M, Navarro A. Epigenetic regulation mechanisms of microRNA expression. Biomol Concepts. 2017;8:203-12.

Mulla MJ, Myrtolli K, Potter J, Boeras C, Kavathas $\mathrm{PB}$, Sfakianaki AK, et al. Uric acid induces trophoblast IL-1beta production via the inflammasome: implications for the pathogenesis of preeclampsia. Am J Reprod Immunol. 2011;65:542-8.

Munaut C, Tebache L, Blacher S, Noël A, Nisolle M, Chantraine F. Dysregulated circulating miRNAs in preeclampsia. Biomed Rep. 2016; 5:686-92.

Neves R, Scheel C, Weinhold S, Honisch E, Iwaniuk KM, Trompeter HI, et al. Role of DNA methylation in miR-200c/141 cluster silencing in invasive breast cancer cells. BMC Res Notes. 2010;3:219. 
Novakovic B, Evain-Brion D, Murthi P, Fournier T, Saffery R. Variable DAXX gene methylation is a common feature of placental trophoblast differentiation, preeclampsia, and response to hypoxia. FASEB J. 2017a;31:238092.

Novakovic B, Fournier T, Harris LK, James J, Roberts CT, Yong HEJ, et al. Increased methylation and decreased expression of homeobox genes TLX1, HOXA10 and DLX5 in human placenta are associated with trophoblast differentiation. Sci Rep. 2017b;7:4523.

Padrão NA, Monteiro-Reis S, Torres-Ferreira J, Antunes L, Leça L, Montezuma D, et al. MicroRNA promoter methylation: a new tool for accurate detection of urothelial carcinoma. Br J Cancer. 2017;116:634-9.

Pan Y, Lu F, Xiong P, Pan M, Zhang Z, Lin X, et al. WIPF1 antagonizes the tumor suppressive effect of miR-141/200c and is associated with poor survival in patients with PDAC. J Exp Clin Cancer Res. 2018;37:167.

Pepin G, Gantier MP. microRNA Decay: Refining microRNA Regulatory Activity. Microrna. 2016;5:167-74

Pozzobon T, Goldoni G, Viola A, Molon B. CXCR4 signaling in health and disease. Immunol Lett. 2016;177:6-15.

Quinn KE, Reynolds LP, Grazul-Bilska AT, Borowicz PP, Ashley RL. Placental development during early pregnancy: Effects of embryo origin on expression of chemokine ligand twelve (CXCL12). Placenta. 2016;43:77-80.

Ritchie W. microRNA Target Prediction. Methods Mol Biol. 2017;1513:193-200.

Sahay AS, Jadhav AT, Sundrani DP, Wagh GN, Mehendale SS, Joshi SR. Matrix metalloproteinases-2 (MMP-2) and matrix metallopro- teinases -9 (MMP-9) are differentially expressed in different regions of normal and preeclampsia placentae. J Cell Biochem. 2018; 119:6657-64.

Saji F, Samejima Y, Kamiura S, Sawai K, Shimoya $\mathrm{K}$, Kimura T. Cytokine production in chorioamnionitis. J Reprod Immunol. 2000;47:18596.

Skalis G, Katsi V, Miliou A, Georgiopoulos G, Papazachou O, Vamvakou G, et al. MicroRNAs in Preeclampsia. Microrna. 2019;8:28-35.

Sleightholm RL, Neilsen BK, Li J, Steele MM, Singh RK, Hollingsworth MA, et al. Emerging roles of the CXCL12/CXCR4 axis in pancreatic cancer progression and therapy. Pharmacol Ther. 2017;179:158-70.

Steegers EA, von Dadelszen P, Duvekot JJ, Pijnenborg R. Pre-eclampsia. Lancet. 2010;376:63144.

Stødle GS, Silva GB, Tangerås LH, Gierman LM, Nervik I, Dahlberg UE, et al. Placental inflammation in pre-eclampsia by Nod-like receptor protein (NLRP) 3 inflammasome activation in trophoblasts. Clin Exp Immunol. 2018;193: 84-94.

Sun B, Hu L, Luo ZY, Chen XP, Zhou HH, Zhang W. DNA methylation perspectives in the pathogenesis of autoimmune diseases. Clin Immunol. 2016;164:21-7.

Unamuno X, Gómez-Ambrosi J, Ramírez B, Rodríguez A, Becerril S, Valentí V, et al. NLRP3 inflammasome blockade reduces adipose tissue inflammation and extracellular matrix remodeling. Cell Mol Immunol. 2021;18:104557.

Wang L, Li X, Zhao Y, Fang C, Lian Y, Gou W, et al. Insights into the mechanism of CXCL12mediated signaling in trophoblast functions and placental angiogenesis. Acta Biochim Biophys Sin (Shanghai). 2015;47:663-72.

Wu D, Chen X, Wang L, Chen F, Cen H, Shi L. Hypoxia-induced microRNA-141 regulates trophoblast apoptosis, invasion, and vascularization by blocking CXCL12 $\beta / C X C R 2 / 4$ signal transduction. Biomed Pharmacother. 2019;116:108836.

Wu H, Wang H, Liu M, Bai Y, Li YX, Ji L, et al. MiR-195 participates in the placental disorder of preeclampsia via targeting activin receptor type-2B in trophoblastic cells. J Hypertens. 2016;34:1371-9.

Xu Y, Wu D, Jiang Z, Zhang Y, Wang S, Ma Z, et al. MiR-616-3p modulates cell proliferation and migration through targeting tissue factor pathway inhibitor 2 in preeclampsia. Cell Prolif. 2018;51:e12490.

Xue LJ, Mao XB, Ren LL, Chu XY. Inhibition of CXCL12/CXCR4 axis as a potential targeted therapy of advanced gastric carcinoma. Cancer Med. 2017;6:1424-36.

Yeung KR, Chiu CL, Pidsley R, Makris A, Hennessy A, Lind JM. DNA methylation profiles in preeclampsia and healthy control placentas. Am J Physiol Heart Circ Physiol. 2016; 310:H1295-303.

Zendedel A, Johann S, Mehrabi S, Joghataei MT, Hassanzadeh G, Kipp M, et al. Activation and Regulation of NLRP3 Inflammasome by Intrathecal Application of SDF-1a in a Spinal Cord Injury Model. Mol Neurobiol. 2016;53:3063-75.

Zhang K, Fan C, Cai D, Zhang Y, Zuo R, Zhu L, et al. Contribution of TGF-Beta-Mediated NLRP3-HMGB1 Activation to Tubulointerstitial Fibrosis in Rat with Angiotensin II-Induced Chronic Kidney Disease. Front Cell Dev Biol. 2020;8:1. 